Open J. Math. Sci., Vol. 2(2018), No. 1, pp. 56 - 72

Website: https://pisrt.org/psr-press/journals/oms/

ISSN: 2523-0212 (Online) 2616-4906 (Print)

http://dx.doi.org/10.30538/oms2018.0017

\title{
FIXED POINT RESULTS FOR THE COMPLEX FRACTAL GENERATION IN THE $S$-ITERATION ORBIT WITH $s$-CONVEXITY
}

\author{
KRZYSZTOF GDAWIEC ${ }^{1}$, ABDUL AZIZ SHAHID
}

\begin{abstract}
Since the introduction of complex fractals by Mandelbrot they gained much attention by the researchers. One of the most studied complex fractals are Mandelbrot and Julia sets. In the literature one can find many generalizations of those sets. One of such generalizations is the use of the results from fixed point theory. In this paper we introduce in the generation process of Mandelbrot and Julia sets a combination of the $S$-iteration, known from the fixed point theory, and the $s$-convex combination. We derive the escape criteria needed in the generation process of those fractals and present some graphical examples.

Mathematics Subject Classification: 37F45, 37F50, 47J25

Key words and phrases: itration schemes; Julia set; Mandelbrot set; escape criterion.
\end{abstract}

\section{Introduction}

Mandelbrot and Julia sets are some of the best known illustrations of a highly complicated chaotic systems generated by a very simple mathematical process. They were introduced by Benoit Mandelbrot in the late 1970's [1], but Julia sets were studied much earlier, namely in the early 20th century by French mathematicians Pierre Fatou and Gaston Julia. Mandelbrot working at IBM has studied their works and plotted the Julia sets for $z^{2}+c$ and corresponding to them the Mandelbrot set. He was surprised by the result that he obtained. Since then many mathematicians have studied different properties of Mandelbrot

\footnotetext{
Received 15 November 2017. Revised 29 March 2018.

${ }^{1}$ Corresponding Author

(C) 2018 Krzysztof Gdawiec, Abdul Aziz Shahid. This is an open access article distributed under the Creative Commons Attribution License, which permits unrestricted use, distribution, and reproduction in any medium, provided the original work is properly cited.
} 
and Julia sets and proposed various generalizations of those sets. The first and the most obvious generalization was the use of $z^{p}+c$ function instead of the quadratic one used by Mandelbrot [2,3]. Then some other types of functions were studied: rational [4], transcendental [5], elliptic [6], anti-polynomials [7] etc. Another step in the studies on Mandelbrot and Julia sets was the extension from complex numbers to other algebras, e.g., quaternions [8], octonions [9], bicomplex numbers [10] etc.

Another interesting generalization of Mandelbrot and Julia sets is the use of the results from fixed point theory. In the fixed point theory there exist many approximate methods of finding fixed points of a given mapping, that are based on the use of different feedback iteration processes. These methods can be used in the generalization of Mandelbrot and Julia sets. In 2004, Rani and Kumar $[11,12]$ introduced superior Julia and Mandelbrot sets using Mann iteration scheme. Chauhan et al. in [13] introduced the relative superior Julia sets using Ishikawa iteration scheme. Also, relative superior Julia sets, Mandelbrot sets and tricorn, multicorns by using the $S$-iteration scheme were presented in $[14,15]$. Recently, Ashish et al. in [16] introduced Julia and Mandelbrot sets using the Noor iteration scheme, which is a three-step iterative procedure. The junction of a $s$-convex combination [17] and various iteration schemes was studied in many papers. Mishra et al. $[18,19]$ developed fixed point results in relative superior Julia sets, tricorn and multicorns by using the Ishikawa iteration with $s$-convexity. In [20] Kang et al. introduced new fixed point results for fractal generation using the implicit Jungck-Noor orbit with $s$-convexity, whereas Nazeer et al. in [21] used the Jungck-Mann and Jungck-Ishikawa iterations with $s$-convexity. The use of Noor iteration and $s$-convexity was shown in [22].

In this paper, we present some fixed point results for Julia and Mandelbrot sets by using the $S$-iteration scheme with $s$-convexity. We derive the escape criteria for quadratic, cubic and the $(k+1)$ th degree complex polynomial.

The remainder of this paper is outlined as follows. In Sec. 2 we present some basic definitions used in the paper. Next, in Sec. 3, we present the main results of this paper, namely we prove the escape criteria for the quadratic, cubic and the $(k+1)$ th degree complex polynomial in the $S$-iteration with $s$-convexity. Some graphical examples of complex fractals generated using the escape criteria are presented in Sec. 4. The paper ends with some concluding remarks and future work (Sec. 5).

\section{Preliminaries}

Definition 2.1 (see [23], Julia set). Let $f: \mathbb{C} \rightarrow \mathbb{C}$ be a polynomial of degree $\geq 2$. Let $F_{f}$ be the set of points in $\mathbb{C}$ whose orbits do not converge to the point at infinity, i.e., $F_{f}=\left\{z \in \mathbb{C}:\left\{\left|f^{n}(z)\right|\right\}_{n=0}^{\infty}\right.$ is bounded $\}$. $F_{f}$ is called as filled Julia set of the polynomial $f$. The boundary points of $F_{f}$ are called the points of Julia set of the polynomial $f$ or simply the Julia set. 
Definition 2.2 (see [24], Mandelbrot set). The Mandelbrot set $M$ consists of all parameters $c$ for which the filled Julia set of $Q_{c}(z)=z^{2}+c$ is connected, i.e.,

$$
M=\left\{c \in \mathbb{C}: F_{Q_{c}} \text { is connected }\right\} .
$$

In fact, $M$ contains an enormous amount of information about the structure of Julia sets.

The Mandelbrot set $M$ for the quadratic $Q_{c}(z)=z^{2}+c$ can be equivalently defined in the following way:

$$
M=\left\{c \in \mathbb{C}:\left\{Q_{c}^{n}(0)\right\} \text { does not tend to } \infty \text { as } n \rightarrow \infty\right\},
$$

We choose the initial point 0 , because 0 is the only critical point of $Q_{c}$, i.e., $Q_{c}^{\prime}(0)=0$.

Definition 2.3. Let $C \subset \mathbb{C}$ be a non-empty set and $f: C \rightarrow C$. For any point $z_{0} \in C$ the Picard orbit is defined as the set of iterates of the point $z_{0}$, i.e.,

$$
O\left(f, z_{0}\right)=\left\{z_{n}: z_{n}=f\left(z_{n-1}\right), n=1,2,3, \ldots\right\},
$$

where the orbit $O\left(f, z_{0}\right)$ of $f$ at the initial point $z_{0}$ is the sequence $\left\{f^{n}\left(z_{0}\right)\right\}_{n=1}^{\infty}$.

Definition 2.4 (see [25], $S$-iteration orbit). Consider a sequence $\left\{z_{n}\right\}$ of iterates for initial point $z_{0} \in C$ such that

$$
\left\{\begin{array}{l}
z_{n+1}=\left(1-\gamma_{n}\right) f\left(z_{n}\right)+\gamma_{n} f\left(w_{n}\right), \\
w_{n}=\left(1-\delta_{n}\right) z_{n}+\delta_{n} f\left(z_{n}\right),
\end{array}\right.
$$

where $n=0,1,2, \ldots$ and $\gamma_{n}, \delta_{n} \in(0,1]$. This sequence of iterates is called the $S$ orbit, which is a function of four arguments $\left(f, z_{0}, \gamma_{n}, \delta_{n}\right)$ and we will denote it by $S O\left(f, z_{0}, \gamma_{n}, \delta_{n}\right)$.

In [14] Kang et al. have proved the escape criterion for the Mandelbrot and Julia sets in $S$-orbit.

Theorem 2.5 (Escape Criterion for $S$-iteration). Let $Q_{c}(z)=z^{k+1}+c$, where $k=1,2,3, \ldots$ and $c \in \mathbb{C}$. Iterate $Q_{c}$ using (4) with $\gamma_{n}=\gamma, \delta_{n}=\delta$, where $\gamma, \delta \in(0,1]$. Suppose that

$$
|z|>\max \left\{|c|,\left(\frac{2}{\gamma}\right)^{\frac{1}{k}},\left(\frac{2}{\delta}\right)^{\frac{1}{k}}\right\},
$$

then there exist $\lambda>0$ such that $\left|z_{n}\right|>(1+\lambda)^{n}|z|$ and $\left|z_{n}\right| \rightarrow \infty$ as $n \rightarrow \infty$.

\section{Main results}

In each of the two steps of $S$-iteration we use a convex combination of two elements. In the literature we can find some generalizations of the convex combination. One of such generalizations is the $s$-convex combination. 
Definition 3.1 ( $s$-convex combination [17]). Let $z_{1}, z_{2}, \ldots, z_{n} \in \mathbb{C}$ and $s \in$ $(0,1]$. The $s$-convex combination is defined in the following way:

$$
\lambda_{1}^{s} z_{1}+\lambda_{2}^{s} z_{2}+\ldots+\lambda_{n}^{s} z_{n}
$$

where $\lambda_{k} \geq 0$ for $k \in\{1,2, \ldots, n\}$ and $\sum_{k=1}^{n} \lambda_{k}=1$.

Let us notice that the $s$-convex combination for $s=1$ reduces to the standard convex combination. Now, we will replace the convex combination in the $S$ iteration with the $s$-convex one.

Let $Q_{c}$ be a polynomial and $z_{0} \in \mathbb{C}$. We define the $S$-iteration with $s$-convexity as follows:

$$
\left\{\begin{array}{l}
z_{n+1}=(1-\gamma)^{s} Q_{c}\left(z_{n}\right)+\gamma^{s} Q_{c}\left(w_{n}\right), \\
w_{n}=(1-\delta)^{s} z_{n}+\delta^{s} Q_{c}\left(z_{n}\right),
\end{array}\right.
$$

where $n=0,1,2, \ldots$ and $\gamma, \delta, s \in(0,1]$. We will denote the $S$-iteration with $s$-convexity by $S O_{s}\left(f, z_{0}, \gamma, \delta, s\right)$.

In the following subsections we prove escape criteria for some classes of polynomials using the $S$-iteration with $s$-convexity.

\subsection{Escape criterion for quadratic function.}

Theorem 3.2. Assume that $|z| \geq|c|>\frac{2}{s \gamma}$ and $|z| \geq|c|>\frac{2}{s \delta}$, where $\gamma, \delta, s \in$ $(0,1]$ and $c \in \mathbb{C}$. Let $z_{0}=z$. Then for (7) with $Q_{c}(z)=z^{2}+c$ we have $\left|z_{n}\right| \rightarrow \infty$ as $n \rightarrow \infty$.

Proof. Consider

For $Q_{c}(z)=z^{2}+c$

$$
|w|=\left|(1-\delta)^{s} z+\delta^{s} Q_{c}(z)\right|
$$

$$
\begin{aligned}
|w| & =\left|(1-\delta)^{s} z+\delta^{s}\left(z^{2}+c\right)\right| \\
& =\left|(1-\delta)^{s} z+(1-(1-\delta))^{s}\left(z^{2}+c\right)\right| .
\end{aligned}
$$

By binomial expansion upto linear terms of $\delta$ and $(1-\delta)$, we obtain

$$
\begin{aligned}
|w| & \geq\left|(1-s \delta) z+(1-s(1-\delta))\left(z^{2}+c\right)\right| \\
& =\left|(1-s \delta) z+(1-s+s \delta)\left(z^{2}+c\right)\right| \\
& \geq\left|(1-s \delta) z+s \delta\left(z^{2}+c\right)\right|, \text { because } 1-s+s \delta \geq s \delta \\
& \geq\left|s \delta z^{2}+(1-s \delta) z\right|-|s \delta c| \\
& \geq\left|s \delta z^{2}+(1-s \delta) z\right|-|s \delta z|, \text { because }|z| \geq|c| \\
& \geq\left|s \delta z^{2}\right|-|(1-s \delta) z|-|s \delta z| \\
& \geq\left|s \delta z^{2}\right|-|z|+|s \delta z|-|s \delta z| \\
& =|z|(s \delta|z|-1) .
\end{aligned}
$$

In the second step of the $S$-iteration with $s$-convexity we have

$$
\begin{aligned}
\left|z_{1}\right| & =\left|(1-\gamma)^{s} Q_{c}(z)+\gamma^{s} Q_{c}(w)\right| \\
& =\left|(1-\gamma)^{s}\left(z^{2}+c\right)+(1-(1-\gamma))^{s}\left(w^{2}+c\right)\right| .
\end{aligned}
$$


By binomial expansion upto linear terms of $\gamma$ and $(1-\gamma)$, we obtain

$$
\begin{aligned}
\left|z_{1}\right| & \geq\left|(1-s \gamma)\left(z^{2}+c\right)+(1-s(1-\gamma))\left(w^{2}+c\right)\right| \\
& =\left|(1-s \gamma)\left(z^{2}+c\right)+(1-s+s \gamma)\left(w^{2}+c\right)\right| \\
& \geq\left|(1-s \gamma)\left(z^{2}+c\right)+s \gamma\left((|z|(s \delta|z|-1))^{2}+c\right)\right|, \text { because } 1-s+s \gamma \geq(\text { ( } 1 \mathrm{Q})
\end{aligned}
$$

Since $|z|>2 /(s \delta)$, which implies $s \delta|z|>2$ and $(s \delta|z|-1)^{2}>1$. Thus

$$
|z|^{2}(s \delta|z|-1)^{2}>|z|^{2}>\delta|z|^{2} \quad(\because 0<\delta<1)
$$

Using (11) in (10) we have

$$
\begin{aligned}
\left|z_{1}\right| & \geq\left|(1-s \gamma)\left(z^{2}+c\right)+s \gamma\left(\delta|z|^{2}+c\right)\right| \\
& =\left.|s \gamma \delta| z\right|^{2}+(1-s \gamma) z^{2}+(1-s \gamma) c+s \gamma c \mid \\
& =\left.|s \gamma \delta| z\right|^{2}+(1-s \gamma) z^{2}+c \mid \\
& \geq s \gamma \delta|z|^{2}-\left|(s \gamma-1) z^{2}\right|-|c| \\
& \geq s \gamma \delta|z|^{2}-(s \gamma-1)\left|z^{2}\right|-|z|(\because|z|>|c|) \\
& =|z|((s \gamma \delta-s \gamma+1)|z|-1) .
\end{aligned}
$$

Because $|z|>2 /(s \gamma)$ and $|z|>2 /(s \delta)$, so

$$
|z|>\frac{2}{s \gamma \delta}>\frac{2}{s \gamma \delta-s \gamma+1}
$$

which implies

$$
(s \gamma \delta-s \gamma+1)|z|-1>1 .
$$

Therefore, there exists $\lambda>0$, such that $(s \gamma \delta-\gamma+1)|z|-1>1+\lambda>1$. Consequently

$$
\left|z_{1}\right|>(1+\lambda)|z|
$$

We may apply the same argument repeatedly to obtain:

$$
\begin{aligned}
\left|z_{2}\right|> & (1+\lambda)^{2}|z|, \\
& \vdots \\
\left|z_{n}\right|> & (1+\lambda)^{n}|z| .
\end{aligned}
$$

Hence, $\left|z_{n}\right| \rightarrow \infty$ as $n \rightarrow \infty$. This completes the proof.

Corollary 3.3. Suppose that

$$
|c|>\frac{2}{s \gamma} \text { and }|c|>\frac{2}{s \delta},
$$

then the orbit $S O_{s}\left(Q_{c}, 0, \gamma, \delta, s\right)$ escapes to infinity.

The following corollary is the refinement of the escape criterion. 
Corollary 3.4 (Escape Criterion). Let $\gamma, \delta, s \in(0,1]$. Suppose that

$$
|z|>\max \left\{|c|, \frac{2}{s \gamma}, \frac{2}{s \delta}\right\}
$$

then there exist $\lambda>0$ such that $\left|z_{n}\right|>(1+\lambda)^{n}|z|$ and $\left|z_{n}\right| \rightarrow \infty$ as $n \rightarrow \infty$.

\subsection{Escape criterion for cubic function.}

Theorem 3.5. Assume that $|z| \geq|c|>\left(\frac{2}{s \gamma}\right)^{\frac{1}{2}}$ and $|z| \geq|c|>\left(\frac{2}{s \delta}\right)^{\frac{1}{2}}$, where $c \in \mathbb{C}$ and $\gamma, \delta, s \in(0,1]$. Let $z_{0}=z$. Then for (7) with $Q_{c}(z)=z^{3}+c$ we have $\left|z_{n}\right| \rightarrow \infty$ as $n \rightarrow \infty$.

Proof. Consider

$$
|w|=\left|(1-\delta)^{s} z+\delta^{s} Q_{c}(z)\right|
$$

For $Q_{c}(z)=z^{3}+c$, we have

$$
\begin{aligned}
|w| & =\left|(1-\delta)^{s} z+\delta^{s}\left(z^{3}+c\right)\right| \\
& =\left|(1-\delta)^{s} z+(1-(1-\delta))^{s}\left(z^{3}+c\right)\right| .
\end{aligned}
$$

By binomial expansion upto linear terms of $\delta$ and $(1-\delta)$, we obtain

$$
\begin{aligned}
|w| & \geq\left|(1-s \delta) z+(1-s(1-\delta))\left(z^{3}+c\right)\right| \\
& =\left|(1-s \delta) z+(1-s+s \delta)\left(z^{3}+c\right)\right| \\
& \geq\left|(1-s \delta) z+s \delta\left(z^{3}+c\right)\right|, \text { because } 1-s+s \delta \geq s \delta \\
& \geq\left|s \delta z^{3}+(1-s \delta) z\right|-|s \delta c| \\
& \geq\left|s \delta z^{3}+(1-s \delta) z\right|-|s \delta z|, \text { because }|z| \geq|c| \\
& \geq\left|s \delta z^{3}\right|-|(1-s \delta) z|-|s \delta z| \\
& \geq\left|s \delta z^{3}\right|-|z|+|s \delta z|-|s \delta z| \\
& =|z|\left(s \delta|z|^{2}-1\right) .
\end{aligned}
$$

In the second step of the $S$-iteration with $s$-convexity we have

$$
\begin{aligned}
\left|z_{1}\right| & =\left|(1-\gamma)^{s} Q_{c}(z)+\gamma^{s} Q_{c}(w)\right| \\
& =\left|(1-\gamma)^{s}\left(z^{3}+c\right)+(1-(1-\gamma))^{s}\left(w^{3}+c\right)\right| .
\end{aligned}
$$

By binomial expansion upto linear terms of $\gamma$ and $(1-\gamma)$, we obtain

$$
\begin{aligned}
\left|z_{1}\right| & \geq\left|(1-s \gamma)\left(z^{3}+c\right)+(1-s(1-\gamma))\left(w^{3}+c\right)\right| \\
& =\left|(1-s \gamma)\left(z^{3}+c\right)+(1-s+s \gamma)\left(w^{3}+c\right)\right| \\
& \geq\left|(1-s \gamma)\left(z^{3}+c\right)+s \gamma\left(\left(|z|\left(s \delta|z|^{2}-1\right)\right)^{3}+c\right)\right|, \text { because } 1-s+s \gamma \geq(16)
\end{aligned}
$$

Since $|z|>(2 /(s \delta))^{\frac{1}{2}}$, which implies $s \delta|z|^{2}>2$ and $\left(s \delta|z|^{2}-1\right)^{3}>1$. Thus

$$
|z|^{3}\left(s \delta|z|^{2}-1\right)^{3}>|z|^{3}>\delta|z|^{3} \quad(\because 0<\delta<1) .
$$

Using (17) in (16) we have

$$
\left|z_{1}\right| \geq\left|(1-s \gamma)\left(z^{3}+c\right)+s \gamma\left(\delta|z|^{3}+c\right)\right|
$$




$$
\begin{aligned}
& =\left.|s \gamma \delta| z\right|^{3}+(1-s \gamma) z^{3}+(1-s \gamma) c+s \gamma c \mid \\
& =\left.|s \gamma \delta| z\right|^{3}+(1-s \gamma) z^{3}+c \mid \\
& \geq s \gamma \delta|z|^{3}-\left|(s \gamma-1) z^{3}\right|-|c| \\
& \geq s \gamma \delta|z|^{3}-(s \gamma-1)\left|z^{3}\right|-|z|(\because|z|>|c|) \\
& \geq|z|\left((s \gamma \delta-s \gamma+1)|z|^{2}-1\right) .
\end{aligned}
$$

Because $|z|>(2 /(s \gamma))^{\frac{1}{2}}$ and $|z|>(2 /(s \delta))^{\frac{1}{2}}$, so

$$
|z|^{2}>\frac{2}{s \gamma \delta}>\frac{2}{s \gamma \delta-s \gamma+1}
$$

which implies

$$
(s \gamma \delta-s \gamma+1)|z|^{2}-1>1 .
$$

Therefore, there exists $\lambda>0$, such that $(s \gamma \delta-\gamma+1)|z|^{2}-1>1+\lambda>1$. Consequently

$$
\left|z_{1}\right|>(1+\lambda)|z|
$$

We may apply the same argument repeatedly to obtain:

$$
\begin{aligned}
\left|z_{2}\right|> & (1+\lambda)^{2}|z|, \\
& \vdots \\
\left|z_{n}\right|> & (1+\lambda)^{n}|z| .
\end{aligned}
$$

Hence $\left|z_{n}\right| \rightarrow \infty$ as $n \rightarrow \infty$. This completes the proof.

Corollary 3.6. Suppose that

$$
|c|>\left(\frac{2}{s \gamma}\right)^{\frac{1}{2}} \text { and }|c|>\left(\frac{2}{s \delta}\right)^{\frac{1}{2}}
$$

then the orbit $S O_{s}\left(Q_{c}, 0, \gamma, \delta, s\right)$ escapes to infinity.

The following corollary is the refinement of the escape criterion.

Corollary 3.7 (Escape Criterion). Let $\gamma, \delta, s \in(0,1]$. Suppose that

$$
|z|>\max \left\{|c|,\left(\frac{2}{s \gamma}\right)^{\frac{1}{2}},\left(\frac{2}{s \delta}\right)^{\frac{1}{2}}\right\},
$$

then there exist $\lambda>0$ such that $\left|z_{n}\right|>(1+\lambda)^{n}|z|$ and $\left|z_{n}\right| \rightarrow \infty$ as $n \rightarrow \infty$. 


\subsection{A general escape criterion.}

Theorem 3.8. Assume that $|z| \geq|c|>\left(\frac{2}{s \gamma}\right)^{\frac{1}{k+1}}$ and $|z| \geq|c|>\left(\frac{2}{s \delta}\right)^{\frac{1}{k+1}}$, where $k=1,2, \ldots, c \in \mathbb{C}$ and $\gamma, \delta, s \in(0,1]$. Let $z_{0}=z$. Then for (7) with $Q_{c}(z)=z^{k+1}+c$ we have $\left|z_{n}\right| \rightarrow \infty$ as $n \rightarrow \infty$.

Proof. Consider

$$
|w|=\left|(1-\delta)^{s} z+\delta^{s} Q_{c}(z)\right|
$$

For $Q_{c}(z)=z^{k+1}+c$, we have

$$
\begin{aligned}
|w| & =\left|(1-\delta)^{s} z+\delta^{s}\left(z^{k+1}+c\right)\right| \\
& =\left|(1-\delta)^{s} z+(1-(1-\delta))^{s}\left(z^{k+1}+c\right)\right| .
\end{aligned}
$$

By binomial expansion upto linear terms of $\delta$ and $(1-\delta)$, we obtain

$$
\begin{aligned}
|w| & \geq\left|(1-s \delta) z+(1-s(1-\delta))\left(z^{k+1}+c\right)\right| \\
& =\left|(1-s \delta) z+(1-s+s \delta)\left(z^{k+1}+c\right)\right| \\
& \geq\left|(1-s \delta) z+s \delta\left(z^{k+1}+c\right)\right|, \text { because } 1-s+s \delta \geq s \delta \\
& \geq\left|s \delta z^{k+1}+(1-s \delta) z\right|-|s \delta c| \\
& \geq\left|s \delta z^{k+1}+(1-s \delta) z\right|-|s \delta z|, \text { because }|z| \geq|c| \\
& \geq\left|s \delta z^{k+1}\right|-|(1-s \delta) z|-|s \delta z| \\
& \geq\left|s \delta z^{k+1}\right|-|z|+|s \delta z|-|s \delta z| \\
& =|z|\left(s \delta|z|^{k}-1\right) .
\end{aligned}
$$

In the second step of the $S$-iteration with $s$-convexity we have

$$
\begin{aligned}
\left|z_{1}\right| & =\left|(1-\gamma)^{s} Q_{c}(z)+\gamma^{s} Q_{c}(w)\right| \\
& =\left|(1-\gamma)^{s}\left(z^{k+1}+c\right)+(1-(1-\gamma))^{s}\left(w^{k+1}+c\right)\right| .
\end{aligned}
$$

By binomial expansion upto linear terms of $\gamma$ and $(1-\gamma)$, we obtain

$$
\begin{aligned}
\left|z_{1}\right| & \geq\left|(1-s \gamma)\left(z^{k+1}+c\right)+(1-s(1-\gamma))\left(w^{k+1}+c\right)\right| \\
& =\left|(1-s \gamma)\left(z^{k+1}+c\right)+(1-s+s \gamma)\left(w^{k+1}+c\right)\right| \\
& \geq\left|(1-s \gamma)\left(z^{k+1}+c\right)+s \gamma\left(\left(|z|\left(s \delta|z|^{k}-1\right)\right)^{k+1}+c\right)\right|, \text { since } 1-s+s \gamma \geq(\mathbf{z} 2)
\end{aligned}
$$

Since $|z|>(2 /(s \delta))^{\frac{1}{k}}$, which implies $s \delta|z|^{k}>2$ and $\left(s \delta|z|^{k}-1\right)^{k+1}>1$. Thus

$$
|z|^{k+1}\left(s \delta|z|^{k}-1\right)^{k+1}>|z|^{k+1}>\delta|z|^{k+1} \quad(\because 0<\delta<1) .
$$

Using (23) in (22) we have

$$
\begin{aligned}
\left|z_{1}\right| & \geq\left|(1-s \gamma)\left(z^{k+1}+c\right)+s \gamma\left(\delta|z|^{k+1}+c\right)\right| \\
& =\left.|s \gamma \delta| z\right|^{k+1}+(1-s \gamma) z^{k+1}+(1-s \gamma) c+s \gamma c \mid \\
& =\left.|s \gamma \delta| z\right|^{k+1}+(1-s \gamma) z^{k+1}+c \mid
\end{aligned}
$$




$$
\begin{aligned}
& \geq s \gamma \delta|z|^{k+1}-\left|(s \gamma-1) z^{k+1}\right|-|c| \\
& \geq s \gamma \delta|z|^{k+1}-(s \gamma-1)\left|z^{k+1}\right|-|z|(\because|z|>|c|) \\
& =|z|\left((s \gamma \delta-s \gamma+1)|z|^{k}-1\right) .
\end{aligned}
$$

Because $|z|>(2 /(s \gamma))^{\frac{1}{k}}$ and $|z|>(2 /(s \delta))^{\frac{1}{k}}$, so

$$
|z|^{k}>\frac{2}{s \gamma \delta}>\frac{2}{s \gamma \delta-s \gamma+1}
$$

which implies

$$
(s \gamma \delta-s \gamma+1)|z|^{k}-1>1 .
$$

Therefore, there exists $\lambda>0$, such that $(s \gamma \delta-\gamma+1)|z|^{k}-1>1+\lambda>1$. Consequently

$$
\left|z_{1}\right|>(1+\lambda)|z|
$$

We may apply the same argument repeatedly to obtain:

$$
\begin{aligned}
\left|z_{2}\right|> & (1+\lambda)^{2}|z|, \\
& \vdots \\
\left|z_{n}\right|> & (1+\lambda)^{n}|z| .
\end{aligned}
$$

Hence $\left|z_{n}\right| \rightarrow \infty$ as $n \rightarrow \infty$. This completes the proof.

Corollary 3.9 (Escape Criterion). Let $\gamma, \delta, s \in(0,1]$. Suppose that

$$
|z|>\max \left\{|c|,\left(\frac{2}{s \gamma}\right)^{\frac{1}{k}},\left(\frac{2}{s \delta}\right)^{\frac{1}{k}}\right\},
$$

then there exist $\lambda>0$ such that $\left|z_{n}\right|>(1+\lambda)^{n}|z|$ and $\left|z_{n}\right| \rightarrow \infty$ as $n \rightarrow \infty$.

\section{Generation of Mandelbrot and Julia sets}

In this section we present some graphical examples of Mandelbrot and Julia sets. To generate the images we used the escape time algorithms which were implemented in Mathematica 9.0. Pseudocode of the Mandelbrot set generation algorithm is presented in Algorithm 1, whereas Algorithm 2 presents the pseudocode for the Julia set generation algorithm.

4.1. Mandelbrot sets for the quadratic polynomial $Q_{c}(z)=z^{2}+c$. Examples of a quadratic Mandelbrot set, i.e., the set for $Q_{c}(z)=z^{2}+c$, in the $S$ orbit with $s$-convexity are presented in Fig. 1 . The common parameters used to generate the images were the following: $K=50, \gamma=0.7, \delta=0.2$. Whereas, the varying parameters were the following: (a) $A=[-2,0.3] \times[-1.2,1.2], s=0.1$, (b) $A=[-2.4,0.5] \times[-1.5,1.5], s=0.2$, (c) $A=[-2.6,0.5] \times[-1.8,1.8], s=0.3$, (d) $A=[-3,0.9] \times[-2,2], s=0.4$, (e) $A=[-3.5,0.9] \times[-2,2], s=0.5$, (f) $A=[-4,1] \times[-2.5,2.5], s=0.6$, (g) $A=[-4.5,1] \times[-2.7,2.7], s=0.7$, (h) $A=$ $[-6,1] \times[-3,3], s=0.8$, (i) $A=[-6,1] \times[-3,3], s=0.9$, (j) $A=[-6,1] \times[-3,3]$, $s=1.0$. 


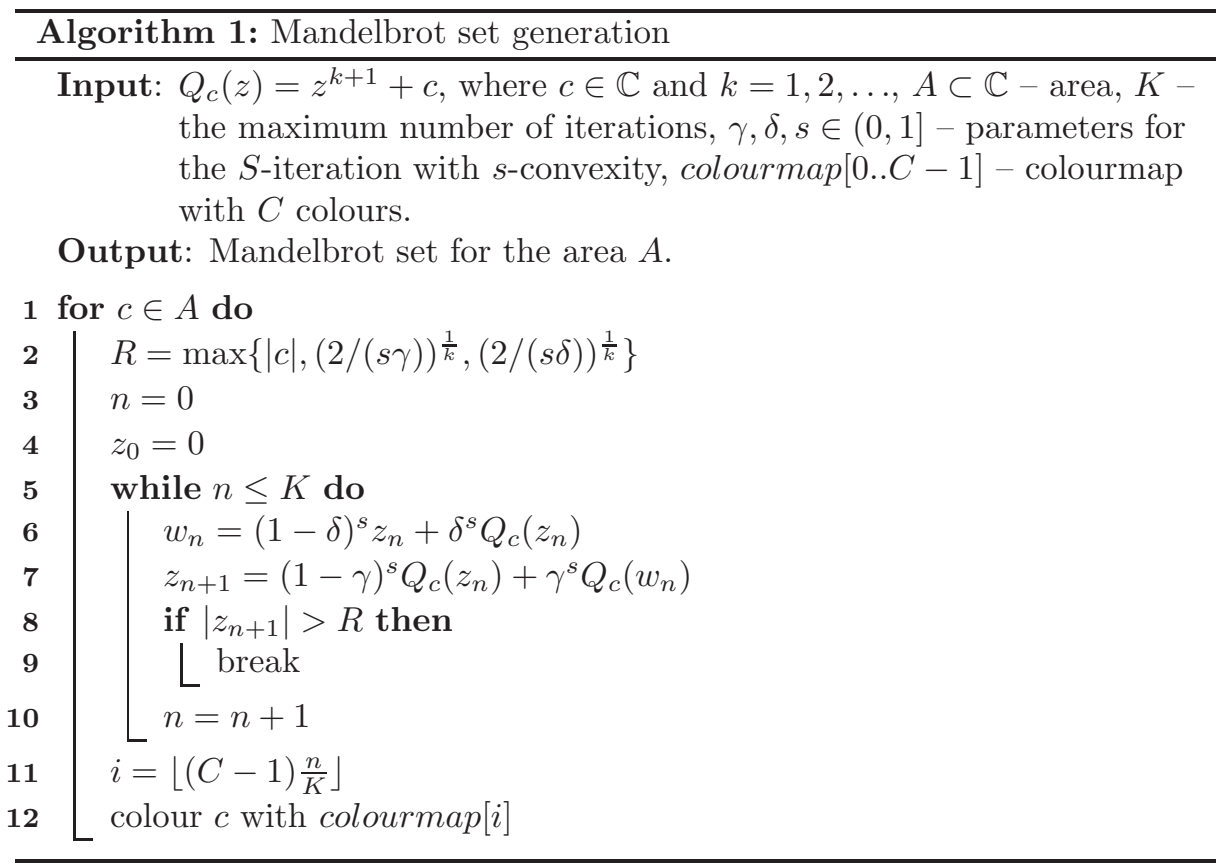

4.2. Mandelbrot sets for the cubic polynomial $Q_{c}(z)=z^{3}+c$. Examples of a cubic Mandelbrot set, i.e., the set for $Q_{c}(z)=z^{3}+c$, in the $S$ orbit with $s$-convexity are presented in Fig. 2. The common parameters used to generate the images were the following: $K=50, \gamma=0.6, \delta=0.4$. Whereas, the varying parameters were the following: (a) $A=[-0.7,0.7] \times[-1,1], s=0.1$, (b) $A=$ $[-0.8,0.8] \times[-1.3,1.3], s=0.2$, (c) $A=[-1,1] \times[-1.5,1.5], s=0.3$, (d) $A=$ $[-1,1] \times[-2,2], s=0.4,(\mathrm{e}) A=[-1,1] \times[-2,2], s=0.5$, (f) $A=[-1,1] \times[-2,2]$, $s=0.6$, (g) $A=[-1,1] \times[-2.2,2.2], s=0.7$, (h) $A=[-1,1] \times[-2.5,2.5], s=0.8$, (i) $A=[-1,1] \times[-2.5,2.5], s=0.9,(\mathrm{j}) A=[-1.5,1.5] \times[-2.5,2.5], s=1.0$.

4.3. Mandelbrot sets for the polynomial $Q_{c}(z)=z^{k+1}+c$, where $k=3$. Examples of the fourth order Mandelbrot set, i.e., the set for $Q_{c}(z)=z^{4}+c$, in the $S$ orbit with $s$-convexity are presented in Fig. 3 . The common parameters used to generate the images were the following: $K=50, \gamma=0.5, \delta=0.3$. Whereas, the varying parameters were the following: (a) $A=[-0.9,0.7] \times$ $[-0.7,0.7], s=0.1$, (b) $A=[-1.1,0.8] \times[-0.8,0.8], s=0.2$, (c) $A=[-1.3,1] \times$ $[-0.9,0.9], s=0.3$, (d) $A=[-1.4,1] \times[-1.1,1.1], s=0.4$, (e) $A=[-1.5,1.1] \times$ $[-1.3,1.3], s=0.5$, (f) $A=[-1.7,1.2] \times[-1.4,1.4], s=0.6$, (g) $A=[-1.8,1.3] \times$ $[-1.5,1.5], s=0.7$, (h) $A=[-1.8,1.4] \times[-1.6,1.6], s=0.8$, (i) $A=[-1.8,1.5] \times$ $[-1.6,1.6], s=0.9,(\mathrm{j}) A=[-1.8,1.5] \times[-1.6,1.6], s=1.0$. 


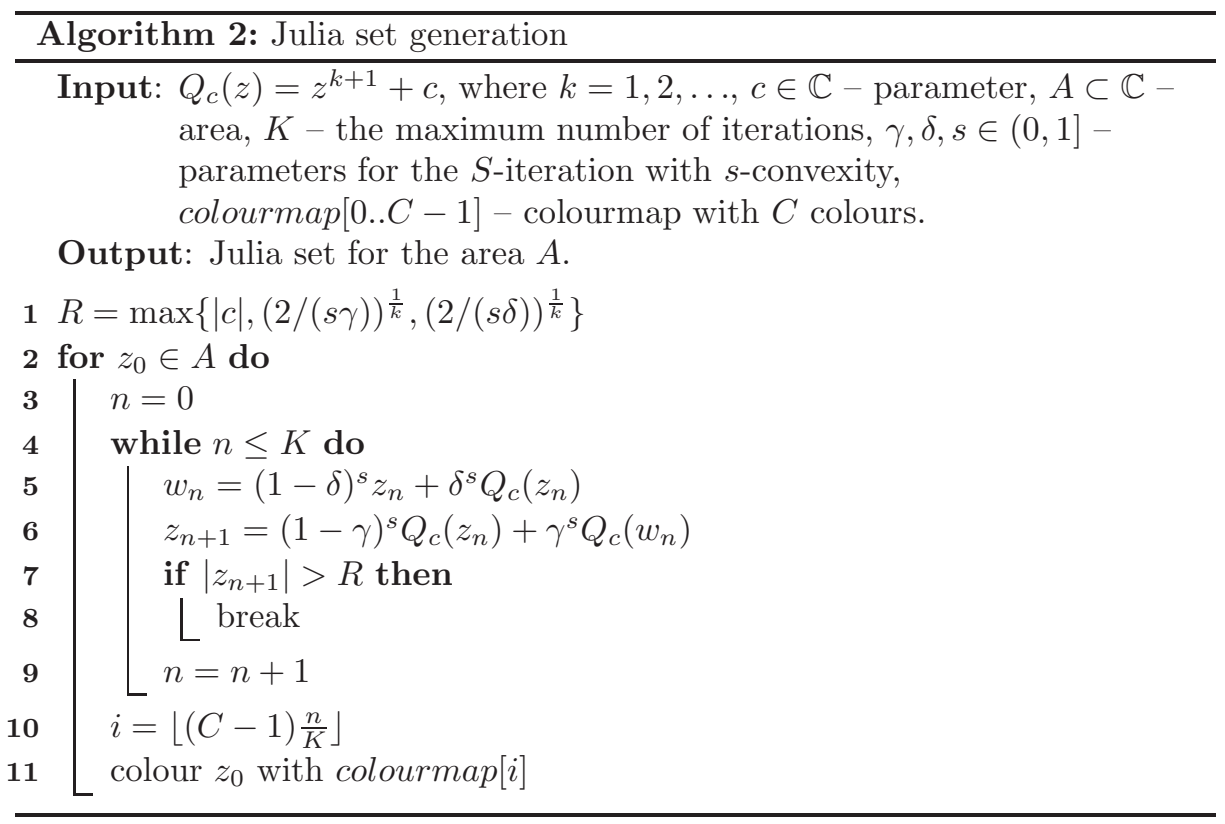

4.4. Julia sets for the quadratic polynomial $Q_{c}(z)=z^{2}+c$. Examples of a quadratic Julia set, i.e., the set for $Q_{c}(z)=z^{2}+c$, in the $S$ orbit with $s$ convexity are presented in Fig. 4. The common parameters used to generate the images were the following: $c=-1.45+0.3 \mathbf{i}, K=50, \gamma=0.3, \delta=0.5$. Whereas, the varying parameters were the following: (a) $A=[-2.1,1.3] \times[-1,1], s=0.1$, (b) $A=[-2.5,1.5] \times[-1.4,1.4], s=0.2$, (c) $A=[-2.3,1.5] \times[-1.4,1.4], s=0.3$, (d) $A=[-2.5,1.7] \times[-1.6,1.6], s=0.4$, (e) $A=[-2.5,1.7] \times[-1.8,1.8], s=0.5$, (f) $A=[-2.5,1.7] \times[-2,2], s=0.6$, (g) $A=[-2.5,1.8] \times[-2.3,2.3], s=0.7$, (h) $A=[-2.5,1.8] \times[-2.6,2.6], s=0.8$, (i) $A=[-2.5,1.8] \times[-2.9,2.9], s=0.9$, (j) $A=[-2.8,2.3] \times[-3.4,3.4], s=1.0$.

\section{Conclusions}

In this paper we proposed the use instead of a convex combination the $s$-convex combination in the $S$-iteration. Using this modified $S$-iteration we derived escape criteria for the polynomial function of the form $z^{k+1}+c$, where $c \in \mathbb{C}$ and $k=1,2, \ldots$. Moreover, we presented some graphical examples of Mandelbrot and Julia sets generated using the escape time algorithm with the derived escape criteria. Very interesting changes in the Mandelbrot and Julia sets can be observed when $s$ varies from low to high values. Because the $s$-convex combination is a generalization of the convex combination, thus the results presented in this paper are generalization of the results obtained by Kang et al. in [14]. 
In our further work we will try to derive the escape criteria in the $S$-iteration with $s$-convexity for functions of other classes than the polynomial one, e.g., trigonometric. Moreover, in the fixed point literature we can find many different iteration methods that can be used in the study of Julia and Mandelbrot sets. A review of explicit iterations and their dependencies can be found in [26].

\section{Competing Interest}

The authors declare no competing interest.

\section{REFERENCES}

1. Mandelbrot, B. B. (1983). The fractal geometry of nature (Vol. 173). New York: WH freeman.

2. Dhurandhar, S. V., Bhavsar, V. C., \& Gujar, U. G. (1993). Analysis of $z$-plane fractal images from $z \leftarrow z^{\alpha}+c$ for $a<0$. Computers \& Graphics, 17(1), 89-94.

3. Lakhtakia, A., Varadan, V. V., Messier, R., \& Varadan, V. K. (1987). On the symmetries of the Julia sets for the process $z \leftarrow z^{p}+c$. Journal of Physics A: Mathematical and General, 20(11), 3533.

4. Peherstorfer, F., \& Stroh, C. (2001). Connectedness of Julia sets of rational functions. Computational Methods and Function Theory, 1(1), 61-79. https://doi.org/10.1007/BF03320977

5. Domnguez, P., \& Fagella, N. (2008, January). Residual Julia sets of rational and transcendental functions. In Transcendental dynamics and complex analysis (pp. 138-164). https://doi.org/10.1017/CBO9780511735233.008

6. Koss, L. (2016). Elliptic Functions with Disconnected Julia Sets. Int. J. Bifurcation Chaos,26 (6), 1650095 (2016)[10 pages].https://doi.org/10.1142/S0218127416500954

7. Crowe, W. D., Hasson, R., Rippon, P. J., \& Strain-Clark, P. E. D. (1989). On the structure of the Mandelbar set. Nonlinearity, 2(4), 541. https://doi.org/10.1088/0951-7715/2/4/003

8. Dang, Y., Kauffman, L. H., \& Sandin, D. J. (2002). Hypercomplex Iterations: Distance Estimation and Higher Dimensional Fractals (Vol. 1). World Scientific. ISBN: 978-981-023296-2

9. Griffin, C. J., \& Joshi, G. C. (1992). Octonionic Julia sets. Chaos, Solitons \& Fractals, 2(1), 11-24. https://doi.org/10.1016/0960-0779(92)90044-N

10. Katunin, A. (2016, September). Analysis of 4D Hypercomplex Generalizations of Julia Sets. In International Conference on Computer Vision and Graphics (pp. 627-635). Springer, Cham.

11. Rani, M., \& Kumar, V. (2004). Superior Julia set. Research in Mathematical Education, 8(4), 261-277.

12. Rani, M., \& Kumar, V. (2004). Superior Mandelbrot set. Research in Mathematical Education, 8(4), 279-291.

13. Chauhan, Y. S., Rana, R., \& Negi, A. (2010). New Julia sets of Ishikawa iterates. International Journal of Computer Applications, 7(13), 34-42.

14. Kang, S. M., Rafiq, A., Latif, A., Shahid, A. A., \& Ali, F. (2016). Fractals through Modified Iteration Scheme. Filomat, 30(11), 3033-3046. https://doi.org/10.2298/FIL1611033K

15. Kang, S. M., Rafiq, A., Latif, A., Shahid, A. A., \& Kwun, Y. C. (2015). Tricorns and Multicorns of-Iteration Scheme. Journal of Function Spaces, Volume 2015, Article ID 417167, 7 pages. http://dx.doi.org/10.1155/2015/417167

16. Rani, M., \& Chugh, R. (2014). Julia sets and Mandelbrot sets in Noor orbit. Applied Mathematics and Computation, 228, 615-631. https://doi.org/10.1016/j.amc.2013.11.077

17. Pinheiro, M. R. (2008). S-convexity (Foundations for Analysis). Differ. Geom. Dyn. Syst, $10,257-262$. 
18. Mishra, M. K., Ojha, D. B., \& Sharma, D. (2011). Fixed point results in tricorn and multicorns of Ishikawa iteration and s-convexity. IJEST, 2(2), 157-160.

19. Mishra, M. K., \& Ojha, D. B. Some Common Fixed Point Results In Relative Superior Julia Sets With Ishikawa Iteration And S-Convexity. IJAEST2(2), 174 - 179

20. Kang, S. M., Nazeer, W., Tanveer, M., \& Shahid, A. A. (2015). New Fixed Point Results for Fractal Generation in Jungck Noor Orbit with-Convexity. Journal of Function Spaces, Volume 2015 (2015), Article ID 963016, 7 pages. http://dx.doi.org/10.1155/2015/963016

21. Nazeer, W., Kang, S. M., Tanveer, M., \& Shahid, A. A. (2015). Fixed point results in the generation of Julia and Mandelbrot sets. Journal of Inequalities and Applications, 2015(1), 298. https://doi.org/10.1186/s13660-015-0820-3

22. Cho, S. Y., Shahid, A. A., Nazeer, W., \& Kang, S. M. (2016). Fixed point results for fractal generation in Noor orbit and s-convexity. SpringerPlus, 5(1), 1843. https://doi.org/10.1186/s4006

23. Barnsley, M. F. (1988). Fractals Everywhere, Acad. Press, New York.

24. Devaney, R. L. (1993). A First Course in Chaotic Dynamical Systems: Theory and Experiment., Avalon Publishing,CA 94608-1077 USA.

25. Agarwal, R. P., O Regan, D., \& Sahu, D. R. (2007). Iterative construction of fixed points of nearly asymptotically nonexpansive mappings. Journal of Nonlinear and convex Analysis, 8(1), 61-79.

26. Gdawiec, K., \& Kotarski, W. (2017). Polynomiography for the polynomial infinity norm via Kalantaris formula and nonstandard iterations. Applied Mathematics and Computation, 307, 17-30. http://dx.doi.org/10.1016/j.amc.2017.02.038

\section{Krzysztof Gdawiec}

Institute of Computer Science, University of Silesia, Bȩdzińska 39, 41-200 Sosnowiec, Poland.

e-mail: kgdawiec@ux2.math.us.edu.pl

Abdul Aziz Shahid

Department of Mathematics and Statistics, University of Lahore, Lahore, Pakistan.

e-mail: abdulazizshahid@gmail.com 


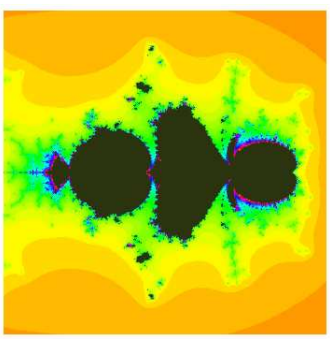

(A) $s=0.1$

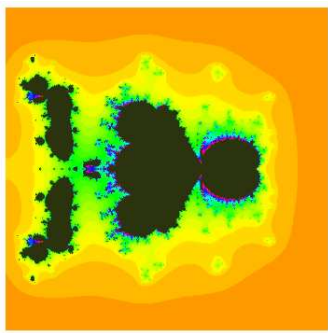

(D) $s=0.4$

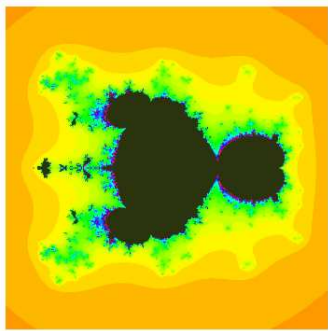

(G) $s=0.7$

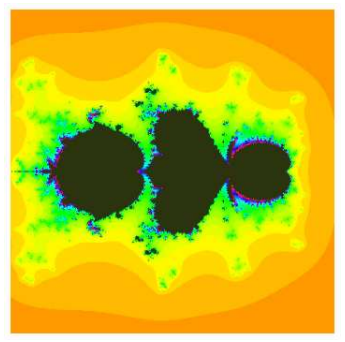

(в) $s=0.2$

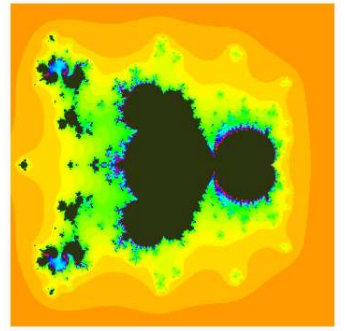

(E) $s=0.5$

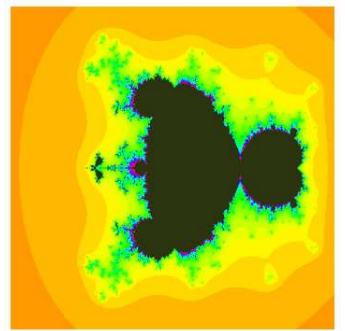

(н) $s=0.8$

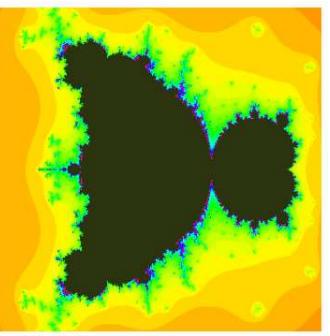

(J) $s=1.0$

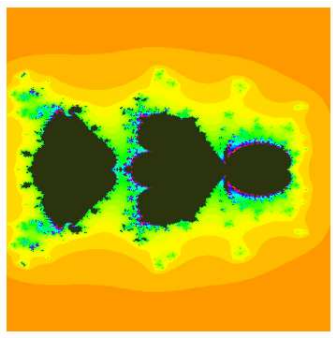

(C) $s=0.3$

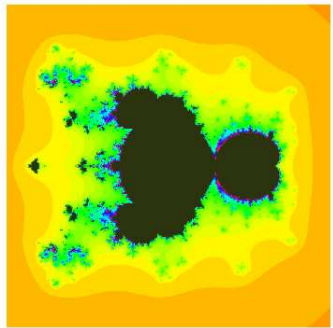

(F) $s=0.6$

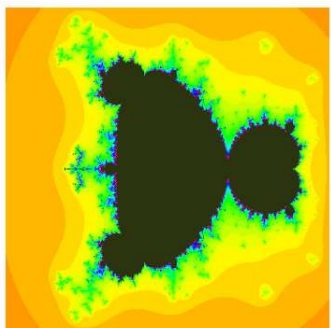

(I) $s=0.9$

Figure 1. Quadratic Mandelbrot set for $\gamma=0.7, \delta=0.2$ and varying $s$ 


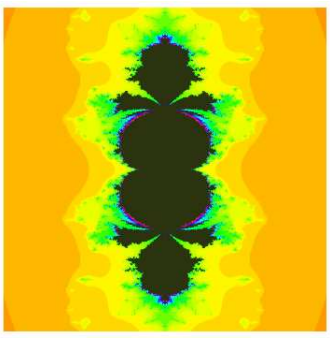

(A) $s=0.1$

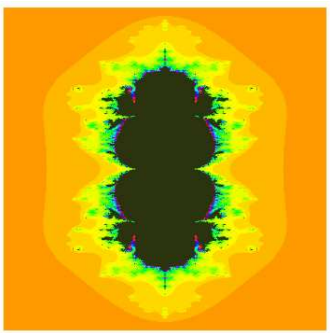

(D) $s=0.4$

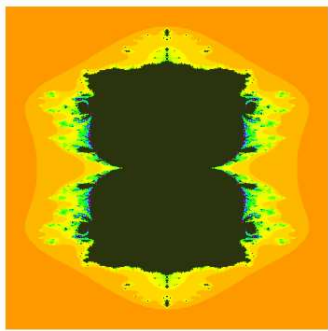

(G) $s=0.7$

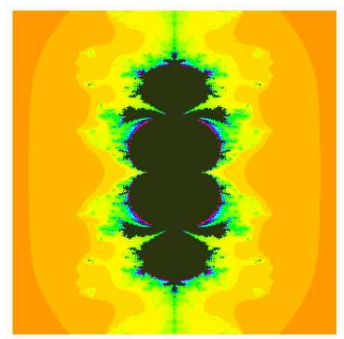

(B) $s=0.2$

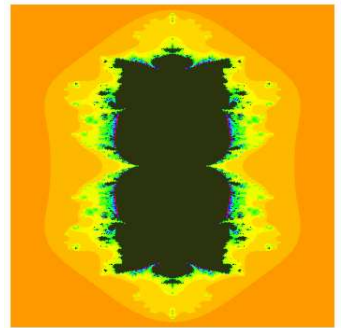

(E) $s=0.5$

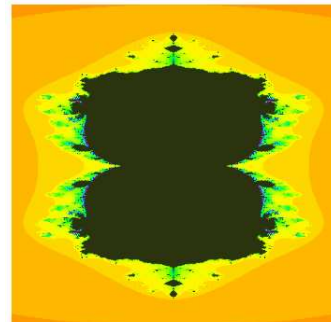

(н) $s=0.8$

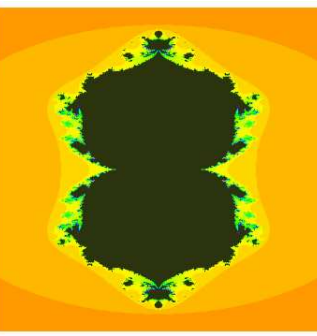

(J) $s=1.0$

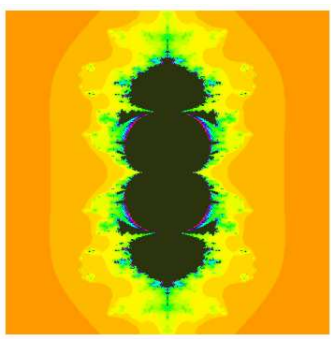

(C) $s=0.3$

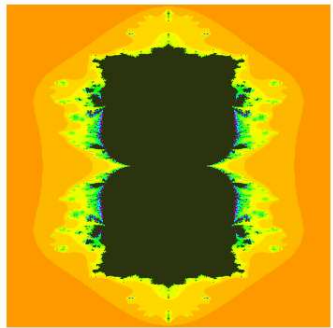

(F) $s=0.6$

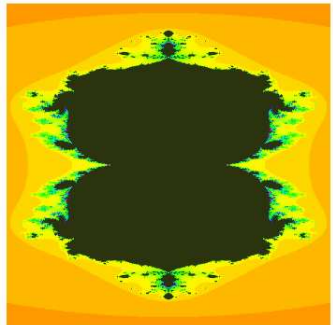

(I) $s=0.9$

Figure 2. Cubic Mandelbrot set for $\gamma=0.6, \delta=0.4$ and varying $s$ 


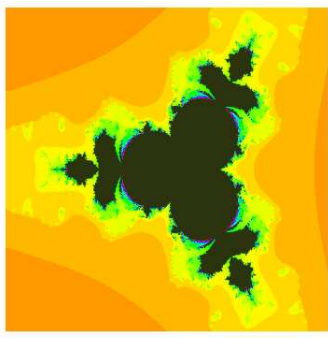

(A) $s=0.1$

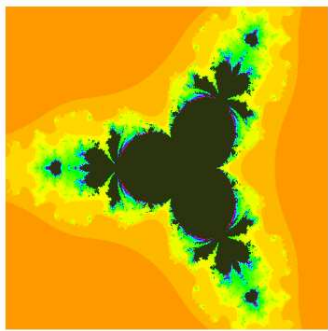

(D) $s=0.4$

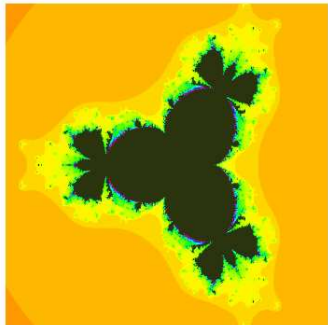

(G) $s=0.7$

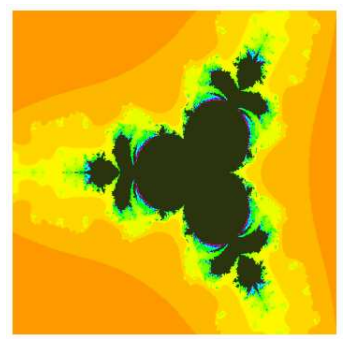

(B) $s=0.2$

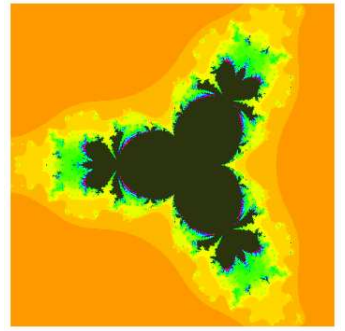

(E) $s=0.5$

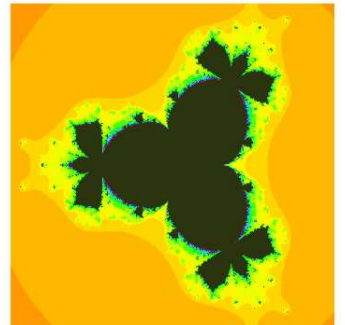

(н) $s=0.8$

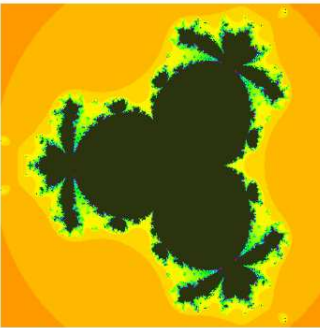

(J) $s=1.0$

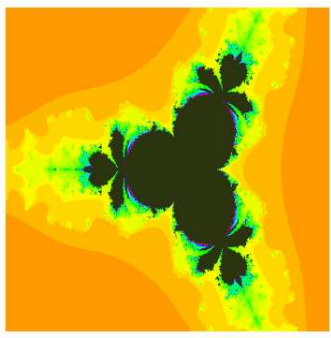

(C) $s=0.3$

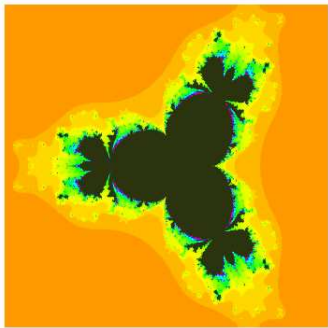

(F) $s=0.6$

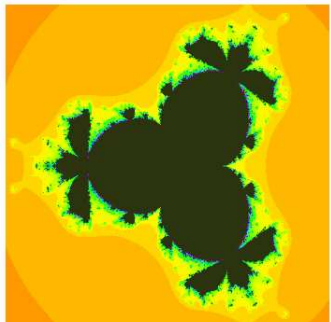

(I) $s=0.9$

Figure 3. Mandelbrot set for $k=3, \gamma=0.5, \delta=0.3$ and varying $s$ 


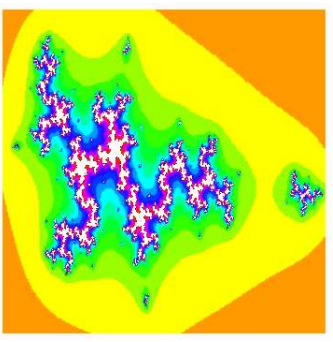

(A) $s=0.1$

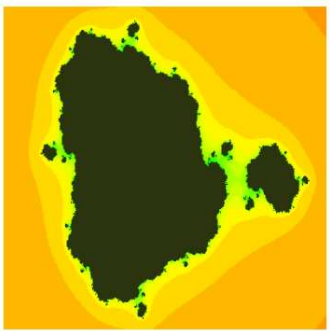

(D) $s=0.4$

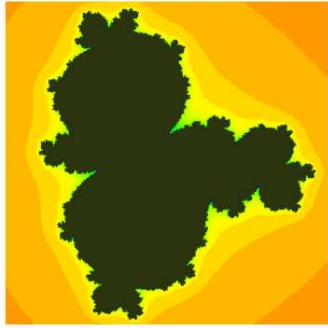

(G) $s=0.7$

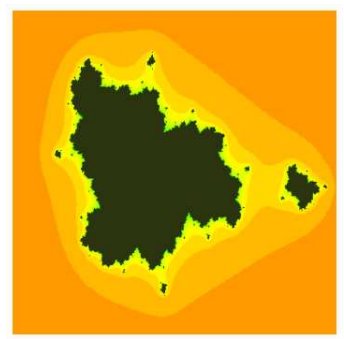

(в) $s=0.2$

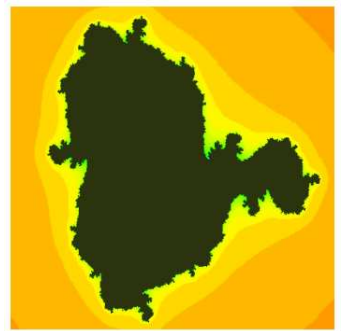

(E) $s=0.5$

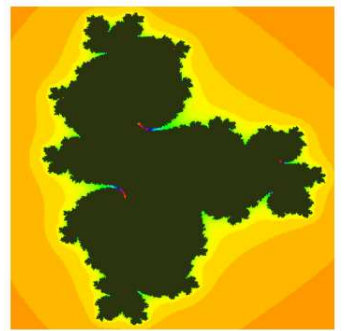

(н) $s=0.8$

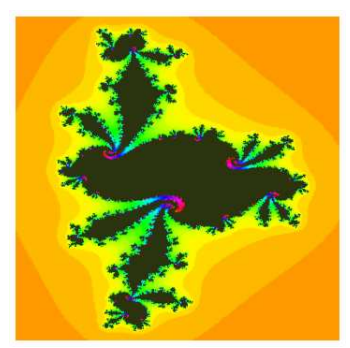

(J) $s=1.0$

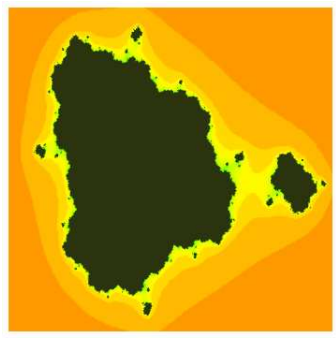

(C) $s=0.3$

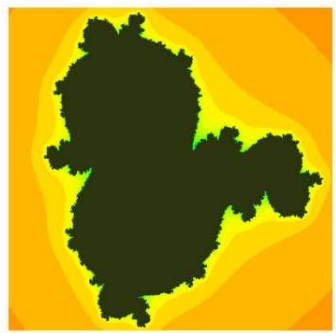

(F) $s=0.6$

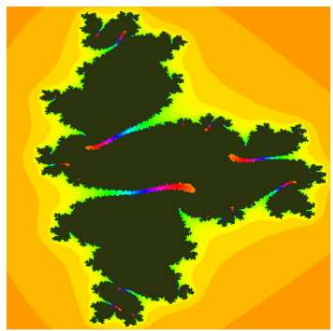

(I) $s=0.9$

Figure 4. Quadratic Julia set for $c=-1.45+0.3 \mathbf{i}, \gamma=0.3, \delta=$ 0.5 and varying $s$ 